\title{
So Long and Thanks for All the EARS: Lessons Learned from Tennessee's Ongoing Syndromic Surveillance Transition
}

\author{
Caleb Wiedeman*, Tonya McKennley, Glenn Yates and Erin Holt \\ Tennessee Department of Health, Nashville, TN, USA
}

\section{Objective}

To share lessons learned in Tennessee during its transition from a jurisdictional syndromic surveillance system to a state-wide, centralized system.

\section{Introduction}

Syndromic surveillance generally refers to the monitoring of disease related events, sets of clinical features (i.e. syndromes), or other indicators in a population. Originally conceived as a tool for the early detection of potential bioterrorism outbreaks, syndromic surveillance is also used by health departments as a tool for monitoring seasonal illness, evaluating health interventions, and other health surveillance activities.

Over the past decade, the Tennessee Department of Health (TDH) has utilized syndromic surveillance at the jurisdictional level. These standalone, jurisdictional systems utilized chief complaint data from local emergency departments (EDs) and the Early Aberration Reporting System (EARS) developed by CDC. Some jurisdictions integrated other local data for analysis in EARS including 911 call center data, over the counter drug sales, and other non-traditional data sources. The analyses conducted on the data varied from jurisdiction to jurisdiction.

CDC dismantled the EARS program in 2011, prompting the need for a complete syndromic surveillance overhaul. TDH decided to implement a centralized, statewide system that would maintain all the capabilities that jurisdictions currently had while allowing for statewide data analysis and aggregation. During this implementation process, TDH has been balancing the short term goal of supporting and maintaining the existing jurisdictional systems while moving forward with acquiring a statewide syndromic surveillance solution and establishing the infrastructure to support it.

\section{Methods}

TDH supports existing EARS systems when possible. Failing EARS systems have been replaced with hybrid systems using Epi Info 7 and SAS. When possible, data transport from hospitals to jurisdictions and TDH is enhanced through automation with scripts written for Microsoft Outlook and WinSCP (a free SFTP client).

TDH is acquiring ESSENCE to serve as a long term syndromic surveillance solution. The ED component of the system will be supported with standardized, HL7 messages received through TDH's electronic data interchange. Large hospital networks are being targeted to pilot the sending of syndromic surveillance messages until TDH declares readiness to receive syndromic surveillance messages for Meaningful Use in October of 2015.

\section{Results}

Maintenance and enhancement of the existing jurisdictional systems has progressed well. Although some of the solutions are not ideal, they have allowed for continued surveillance activities in jurisdictions. Raw, non-standard data from the hybrid systems will be aggregated using SAS and integrated into ESSENCE once available.
TDH's acquisition of ESSENCE has been an arduous process. Administrative delays in the contract and information technology approval process resulted in nearly a year and a half internal review. The HL7 onboarding of hospitals has been a slow process and hospitals and their vendors often require long periods of time to respond to requests for necessary changes.

Many jurisdictions in Tennessee revisited their data use agreements (DUAs) and memorandums of understanding (MOUs) with local hospitals to ensure appropriate permissions and data elements were being provided. Not all of the existing DUAs and MOUs were appropriate for a statewide system and many agreements had to be rewritten. Communications with hospitals have become confused in some instances due to disconnect between the technology departments, traditional public health contacts, and members of hospital staff.

\section{Conclusions}

Moving from a jurisdictional syndromic system to a statewide, centralized syndromic surveillance system is a slow moving and time consuming process. Administrative delays have hindered our ability to get a solution in place rapidly and have resulted in additional work to reproduce failed systems.

Although aggregation and transport of existing non-standard data is possible, we would only recommend it for hospitals that do not have infrastructure for HL7 messaging. Centrally received HL7 messaging makes the data transport process much more efficient and removes the technical burden of receiving and constantly validating data files from our jurisdictional health departments and local hospital staff.

Clear and unified communication among regional and state health departments with hospitals and their corporate leadership is crucial in avoiding confusion and efficiently moving any agreement process forward.

\section{Keywords}

Surveillance system; Tennessee; Syndromic surveillance; Lessons learned

*Caleb Wiedeman
E-mail: caleb.wiedeman@tn.gov 